\title{
Reactive adsorption of penicillin on activated carbons
}

\author{
Conchi O. Ania ${ }^{1,2^{*}}$, Joaquina G. Pelayo ${ }^{1}$, Teresa J. Bandosz ${ }^{2 *}$ \\ ${ }^{1}$ Instituto Nacional del Carbón, CSIC, P.O. 73, 33080 Oviedo, Spain \\ ${ }^{2}$ Chemistry Department of Chemistry, The City College of New York and The Graduate \\ School of CUNY, 160 Convent Ave., New York, NY 10031, USA \\ * Corresponding author email: conchi@incar.csic.es (CO Ania; Tel.: +34 985118846; \\ Fax +34 985297662
}

\begin{abstract}
A series of activated carbons with varied surface chemistry, obtained by wet oxidation and thermal treatment, was used for the removal of penicillin from low concentration aqueous solution. It was found that the carbon surface chemistry favors the degradation of the antibiotic, giving rise to various intermediates detected both in solution and in the adsorbed phase (deposited with the pore structure of the activated carbons). The confinement of penicillin molecules entrapped in the nanopores of activated carbons of acidic nature accelerates their degradation compared to that one in the bulk solution, which can be linked the strong local pH fall inside the pores. Degradation also takes place in activated carbons of basic $\mathrm{pH}$, although the nature and partition of the intermediates formed differ from those in the acidic carbons. In both cases most of the breakdown products do not present therapeutic activity.
\end{abstract}

Keywords: activated carbon, reactive adsorption, penicillin, antibiotics 


\section{Introduction}

Since water is an essential resource for life in all ecosystems, a great effort has been made in the past decades to modify the water sector technologies in order to ensure a sustainable management of its resources while protecting their quality. Particularly, special attention is being paid to the potential environmental and public health impact associated to the occurrence of a new threat to water supplies: the so-called emergent pollutants, originated from consumer products and by products used in industrial, agricultural, and human activities (including prescription and non-prescription human and veterinary pharmaceutical compounds and personal care products among most representatives) [Kolpin, 2002; Daughton, 1999; Jones, 2005].

Pharmaceuticals pose a huge risk as they are continually introduced in the environment at trace level due to their high worldwide consumption. Since a considerable amount of the dose taken is not absorbed by the body, a variety of these chemicals -including painkillers, tranquilizers, anti-depressants, antibiotics, birth control pills, chemotherapy agents, etc.,- are finding their way into the environment via human and animal excreta, from disposal into the sewage system, and from landfill leachate that may impact groundwater supplies. However, little is known about the occurrence, fate, synergistic, and long-term effects of these pollutants and their metabolites following their end-use [Hernando, 2006; Al-Ahmad, 1999; Gomez, 2007]. Control of this kind of pollution is very difficult due to the diversity of sources. Conventional wastewater treatments are not always effective to eliminate and/or degrade the majority of pharmaceutical compounds, which have been found to accumulate in drinking water [Hernando, 2006; Al-Ahmad, 1999; Gomez, 2007; Li, 2008]. The enormous diversity of chemical composition of these pollutants in waters requires special treatment technologies for water decontamination, in order to improve the quality of water and treated wastewater before promoting its reutilization. 
Adsorption is a well-established and highly efficient technology for wastewater remediation. Among the different adsorbents, activated carbon is commonly used for the purification of water with low pollutant concentrations. Its well developed porous structure is responsible for physical adsorption, whereas the variety of its surface chemistry allows specific interactions to take place for a given adsorbate/adsorbent system [Bandosz and Ania, 2006]. Driven by the possibility to synthesize carbon materials with controlled structure and chemical features, their use for the removal of pharmaceuticals offers an interesting and simple tool to deal with this new environmental challenge [Chaubal, 2004; Ania, 2005; Aksu, 2005; Robberson, 2006; Mestre, 2007; Choi, 2008; Ruiz, 2010].

In this paper, we report the removal of a pharmaceutical compound (i.e. penicillin) which is largely used in modern industrial livestock operations and human medicine. Particularly large concentrations are used on livestock and poultry farms (where antibiotics typically are fed to healthy animals for disease prevention, long before the diagnosis), which generate an enormous amount of wastewater often containing undigested antibiotics. It is estimated that as much as $80-90 \%$ of all antibiotics given to humans and animals are not fully broken down and eventually pass through, thus being released into the environment.

The aim of this study is to investigate the reactive adsorption of penicillin on modified carbons. The results are analyzed in terms of the composition of the adsorbent and the solution conditions. To attain this goal, a series of carbons with varied surface chemistry -by incorporating oxygen functionalities via wet oxidation and controlled thermal removal of selected groups- was synthesized and their performance towards penicillin removal from unbuffered aqueous solutions was investigated. The stability of penicillin during the adsorption process was explored, and knowledge of the reactive adsorption mechanism that occurs on the carbon surface, particularly the role of the activated carbon features (porosity and composition) was gained. 


\section{Experimental}

\subsection{Materials}

Activated carbon WVA 1100 (Westvaco, woodbased, $\mathrm{H}_{3} \mathrm{PO}_{4}$ activation) was chosen for this study. The sample WO was obtained by wet oxidation of the initial carbon, denoted as $\mathrm{W}$, in a $20 \%$ nitric acid solution (1 gram carbon:10 $\mathrm{cm}^{3}$ oxidizing solution, stirring overnight). The sample was extensively washed in a Soxhlet apparatus to remove water-soluble species and an excess of oxidizing agent. The oxidized carbon was further submitted to a thermal treatment at various temperatures under inert atmosphere for 1 hour in order to partially remove the surface functionalities. The annealed carbons will be denoted as WOHT, where $\mathrm{T}$ represents the temperature of heat treatment (450 or $700{ }^{\circ} \mathrm{C}$ ). Before usage, all the samples were washed in distilled hot water, dried at $60{ }^{\circ} \mathrm{C}$ overnight and kept in a desiccator.

Penicillin G sodium salt, supplied by Sigma Aldrich is the common name of a $\beta$ lactam antibiotic of formula: sodium [2S-(2 $\alpha, 5 \alpha, 6 \beta)]-3,3-$ dimethyl-7-oxo-6(phenylacetamido)-4-thia-1-azabicyclo[3.2.0]heptane-2-carboxylate] $\left(\mathrm{C}_{16} \mathrm{H}_{17} \mathrm{~N}_{2} \mathrm{NaO}_{4} \mathrm{~S}\right)$. For clarity, the structural formula and selected chemical properties of penicillin G are shown in Figure 1. Unless otherwise stated, all penicillin solutions were prepared in distilled water without $\mathrm{pH}$ adjustment, presenting values of $\mathrm{pH}$ around 6 .

\subsection{Characterization of the activated carbons}

Textural characterization was carried out by measuring the $\mathrm{N}_{2}$ adsorption isotherms at $-196^{\circ} \mathrm{C}$. Before the experiments, the samples were outgassed under vacuum at $120^{\circ} \mathrm{C}$ overnight. The $\mathrm{N}_{2}$ isotherms were used to calculate the specific surface area, $\mathrm{S}_{\mathrm{BET}}$, total pore volume, $\mathrm{V}_{\mathrm{T}}$, and pore size distributions which were evaluated by means of the DFT method using a slit pore geometry.

The point of zero charge $\left(\mathrm{pH}_{\mathrm{PZC}}\right)$, which provides information about the acidity/basicity of the carbons, was measured using a modification of the mass-titration 
method by Noh and Schwarz (1989). About $0.25 \mathrm{~g}$ of carbon powder was dispersed in a suitable volume of distilled water and stored with constant stirring at room temperature until equilibrium was attained (ca. 48 h). Typical activated carbon/water ratios employed were $1,2,4,6,8,10,12,14$ and 16 . Once equilibrium had been reached, the $\mathrm{pH}$ value was measured using a glass electrode. Fresh distilled water was then added in order to obtain the next solid/weight fraction. The plateau in the plot of equilibrium $\mathrm{pH}$ versus solid weight fraction corresponded to the $\mathrm{pH}_{\mathrm{PZC}}$ value of the activated carbon.

Thermal analysis was carried out using a thermogravimetric analyzer from Setaram. Experiments were carried out under an argon flow rate of $50 \mathrm{~cm}^{3} \mathrm{~min}^{-1}$, at a heating rate of $10{ }^{\circ} \mathrm{C} \mathrm{min}{ }^{-1}$, up to a final temperature of $900{ }^{\circ} \mathrm{C}$. For each experiment, about $25 \mathrm{mg}$ of a carbon sample was used. A custom made device for TPD-MS was also used to evaluate the surface chemistry. The samples were heated in a silica fused reactor up to $900{ }^{\circ} \mathrm{C}$ at a heating rate of $10^{\circ} \mathrm{C} \mathrm{min}^{-1}$. The analysis was done under high vacuum conditions (below $10^{-5}$ mbar) and the gas phase was continuously monitored by a mass spectrometer. The amount of $\mathrm{CO}$ and $\mathrm{CO}_{2}$ evolved during the TPD experiments were quantified. The oxygen content was evaluated by direct analysis in a LECO CTF-900 analyzer on the dried carbons.

\subsection{Penicillin adsorption from solution}

Penicillin (PEN) adsorption was carried out from unbuffered solutions (ca. pH 6 units) of initial concentration ranging from 5-100 $\mathrm{mg} \mathrm{l}^{-1}$. All the solutions were prepared with ultra-pure water obtained from Milli-Q water purification systems. The stability of penicillin in solution was initially evaluated at different $\mathrm{pH}$ (са. 2, 6 and 10) by monitoring the evolution of the UV spectrum with time. The PEN breakdown in solution was evaluated from the rate of formation of the $320 \mathrm{~nm}$ absorption peak, arising from the spontaneous splitting of the beta-lactam ring to render penicillenic acid (PENI), a tautomeric form of penicillin [De Weck, 1960]. 
To study the adsorption kinetics, $100 \mathrm{ml}$ of PEN solution (initial concentration $100 \mathrm{mg} \mathrm{l}^{-1}$ ) were mixed with ca. $50 \mathrm{mg}$ of activated carbon in glass vials and continuously stirred (100 rpm) at $25^{\circ} \mathrm{C}$ in a thermostatic regulated water bath. Then the samples were filtered and the PEN concentration in the supernatant liquid was measured on a Shimadzu $2501 \mathrm{UV}$-Vis spectrophotometer at the corresponding wavelength. The amount adsorbed was determined according to $Q_{t}=\left(C_{0^{-}} C t\right) V / m$, where $Q_{\mathrm{t}}$ is the amount $\left(\mathrm{mg} \mathrm{g}^{-1}\right)$ adsorbed at time $t, C_{0}$ is the initial concentration $\left(\mathrm{mg} \mathrm{l}^{-1}\right), C_{\mathrm{t}}$ is the concentration at time $t\left(\mathrm{mg} \mathrm{l}^{-1}\right), V$ is the volume (l) of the adsorbate solution and $m$ is the weight (g) of dried carbon. Blank experiments were also carried out to verify that losses by volatilization or adsorption on the flask walls do not occur. All adsorption assays were made in triplicate. The exhausted carbons after PEN adsorption were washed with water to remove the excess of antibiotic, dried at $60{ }^{\circ} \mathrm{C}$ for overnight, and subsequently analyzed. The compositions of PEN solutions after contact with the carbons (adsorption) were evaluated by reverse-phase HPLC in an apparatus equipped with a photodiode array detector. The separation was conducted using a Spherisorb C-18 column with $5 \mu \mathrm{m}$ particle size $(4.0 \mathrm{~mm} \times 125 \mathrm{~mm})$ and a guard precolumn (40. x $4.0 \mathrm{~mm}$ ) of the same material. The mobile phase consisted of water $\mathrm{KH}_{2} \mathrm{PO}_{4}(0.01 \mathrm{M})$-acetonitrile $(80: 20 \mathrm{v} / \mathrm{v})$ and was pumped at a flow rate of $0.7 \mathrm{~mL} \mathrm{~min}^{-1}$. The injection volume was $20 \mu$ for each sample.

\section{RESULTS AND DISCUSSION}

\subsection{Materials Characterization}

Nitrogen adsorption isotherms obtained for the four carbons are presented in Figure 2, along with the main textural parameters (inset) therein calculated. After oxidation (sample WO), a decrease in the surface area and pore volume was observed as a result of the destruction of some thin pore walls and/or the blocking of the pore entrances by oxygen functional groups. Annealing at mild temperature (sample WOH450) did not cause significant changes in the porosity of the oxidized WO carbon, since the nitrogen adsorption isotherms of both samples practically superimpose in the whole range of 
relative pressures (Figure 2). Only a small increase in the micropore volume of WOH450 (compared to carbon WO) was obtained, which might be due to the partial removal of the oxygen functional groups located at the pore entrances. In contrast, annealing at higher temperature (i.e., $700^{\circ} \mathrm{C}$ ) provoked a slight collapse of the carbon structure caused by the evolution of a large amount of volatiles (decomposition of surface groups). As result, the surface area and pore volumes of sample WOH700 decreased.

This modification in the porosity seen by $\mathrm{N}_{2}$ probe was accompanied by a slight increase in the population of narrow micropores determined by $\mathrm{CO}_{2}$ adsorption at $273 \mathrm{~K}$ (data non shown). This effect was more visible for WOH700 and can be linked to the formation of pores (or opening of the existing ones) during the thermal treatment (ca. a kind of internal gasification during the evolution of $\mathrm{CO}$ and $\mathrm{CO}_{2}$ coming from the decomposition of the surface functionalities of the carbon matrix).

The effect of oxidation and thermal annealing on surface chemistry is much more pronounced. The changes in the amount of oxygen groups were evaluated by elemental analysis, determination of the point of zero charge, and by quantification of the thermodesorbed species by TPD-MS (Figure 3). The results are summarized in Table 1. As expected, nitric acid oxidation caused a significant increase in the amount of carboxylic acids (as $\mathrm{CO}_{2}$-evolving groups below $400^{\circ} \mathrm{C}$ ), carboxylic anhydrides ( $\mathrm{CO}$ and $\mathrm{CO}_{2}$ at temperatures close to $\left.500^{\circ} \mathrm{C}\right)$, lactones $\left(\mathrm{CO}_{2}\right.$ appearing at about $\left.600^{\circ} \mathrm{C}\right)$ and phenols and carbonyl/quinones ( $\mathrm{CO}$ at 700 and $800^{\circ} \mathrm{C}$, respectively) [Figueiredo, 1999; Bandosz and Ania, 2006]. The almost negligible intensity of the $\mathrm{m} / \mathrm{z} 30$ signal corresponding to the eventual decomposition of $\mathrm{N}$-containing groups as $\mathrm{NO}_{\mathrm{x}}$, confirmed that only oxygen-containing surface functionalities were created upon nitric acid oxidation. Certain amounts of $\mathrm{CO}_{2}$ releasing groups were also detected in raw carbon $\mathrm{W}$, as it is synthesized by phosphoric acid activation. Annealing at $450{ }^{\circ} \mathrm{C}$ removed the acidic groups $\left(\mathrm{CO}_{2}\right.$-releasing functionalities) whereas after the treatment at higher temperatures only the phenolic and quinone-type groups were desorbed from the carbon surface. Only small amounts of CO-releasing groups being detected at temperatures above $700{ }^{\circ} \mathrm{C}$. 
These data is in good agreement with the increased acidic character of WO (Table 1) and the increase in the $\mathrm{pH}_{\mathrm{PzC}}$ with the temperature of the thermal treatment. As a result of the latter the annealed carbon exhibits surface hydrophobicity ( $\mathrm{pH}_{\mathrm{PZC}}$ ca. 9.2).

\subsection{Stability of penicillin in solution}

Since it is known that penicillin aqueous solutions might decompose under certain conditions [Schwartz, 1965; Arnott, 1995; Gover, 1983], its stability was investigated (Figure 4A). PEN degradation with time was observed to occur to a small extent in the stock aqueous solution (pH ca. 6.5 units) with the appearance of the strong absorption band at $320 \mathrm{~nm}$ (inset in Figure 4) characteristic of penicillenic acid (PENI). Notwithstanding the kinetics of formation of PENI seemed to be rather slow, with a gradual and small increase in the $320 \mathrm{~nm}$ absorbance with time for 5 days. Thereafter, the intensity of this absorption peak decreased steadily (suggesting further hydrolysis of PENI) until it stabilized, likely due to the formation of penicillenic acid disulfide (which is stable at $\mathrm{pH} 6-7$ units) [Arnott, 1995]. The PEN breakdown to PENI in the solution at neutral $\mathrm{pH}$ accounted for a loss lower than $3 \%$ after 72 hours (inset Figure $4 \mathrm{~A}$ ).

\subsection{Penicillin adsorption from solution}

The adsorption ability of the carbons for the retention of penicillin was determined by kinetic experiments (Figure 5 A). Some modifications were observed in the UV spectra of the PEN solution after contact with some of the carbons, with the appearance of new absorption bands pointing to the degradation of penicillin in the presence of the adsorbents (further confirmed by the HPLC analysis of the solution). The spectra of the solution after contact with carbons $\mathrm{W}$ and WOH700 are shown in Figure $4 \mathrm{~B}$, as an example. Absorption bands at 233 and $285 \mathrm{~nm}$ appear from the first $30 \mathrm{~min}$ of contact with carbons $\mathrm{W}$ and $\mathrm{WO}$, which according to the literature can be assigned to penillic and penilloic acids [Schwartz, 1965; Arnott, 1995; Gover, 1983]. Moreover, the characteristic band of PENI at $320 \mathrm{~nm}$ was detected after contact with all the carbons. The intensities of all these bands were higher in the case of WOH450 and WOH700. 
Data in Figure 5 A represents the rate of penicillin disappearance from solution, which cannot be stricto senso considered as the rate of adsorption due to conversion of PEN into other intermediates. In any case, the rate of penicillin removal was found to reach equilibrium after 48-72 hours regardless the nature of the carbon adsorbent, with most of the uptake within the first 24 hours. The rapid adsorption is attributed to the presence of a large network of a transport pores (mesopores) in all the samples. Moreover, no significant changes were observed in the kinetics of adsorption rate after modification of the carbons (oxidation vs thermal annealing), although a somewhat faster kinetics was observed for sample WOH450, likely due to its larger mesopores volume. These results suggest that although some interactions might occur between the oxygen surface groups and the moieties of penicillin, these do not seem to affect the adsorption rate. The fast kinetics also indicates that accessibility restrictions do not apply, which is in good agreement with the molecular dimensions of penicillin (ca. 0.9 x 0.5 x $0.5 \mathrm{~nm}$, estimated from ChemSketch software after 3D optimization) and the fact that the porosity of all the studied carbons is comprised of large micropores (mean micropore size ca. $1.8 \mathrm{~nm}$ ).

Analysis of the solution after penicillin adsorption on the different carbons by HPLC confirmed the presence of three different compounds in the solution, which abundance appeared to be related to the polarity of carbons (Figure 5 B). In fact, hydrophilic W and WO (both display low $\mathrm{pH}_{\mathrm{PZC}}$ ) favor the degradation of PEN with relatively large amounts of intermediates (mostly penillic and penilloic acids) detected in the solution, whereas a much smaller amount of intermediates was measured for WOH700 and WOH450 which are less polar and thus more hydrophobic. Evaluation of the net amount of penicillin adsorbed on the activated carbons is complex, since the breakdown mechanism of this compound proceeds through various independent and simultaneous pathways. However, a rough estimation of the net efficiency for penicillin removal can be made by considering the amount of penicillin transferred from the solution to the adsorbent (Figure 5A), after subtracting the total amount of intermediates detected in solution (Figure 5B). The 
obtained value follows the sequence: WOH700 $>$ WOH450 $>\mathrm{W}>\mathrm{WO}$, showing a increasing trend with the hydrophobic character of the activated carbons.

From a qualitative point of view, these observations are in agreement with the proposed PEN degradation pathway in aqueous solution upon $\mathrm{pH}$ [Schwartz, 1965; Arnott, 1995; Gover, 1983; Deshpande, 2004]. The breakdown of penicillin to PENI has been reported to be favored in acidic medium, although under these conditions PENI is also unstable and rapidly decomposes to penilloic or penillic acids. At alkaline $\mathrm{pH}$, on the other hand, PEN and PENI are both converted initially to penilloic acid (through formation of penicilloic and further decarboxylation).

Quantitatively, the amount of degradation intermediates detected in the solution after contact with the carbons is smaller than that corresponding to PEN breakdown exclusively due to the $\mathrm{pH}$ effect. This seems reasonable as it is expected that some of the degradation compounds will also be adsorbed in the pores of the activated carbons. To corroborate this possibility, aliquots of the carbons after penicillin adsorption were extracted in acetonitrile (solvent chosen to prevent further degradation of penicillin or its derivatives during the extraction) [Moats and Romanowski, 1998] and analyzed by HPLC. The results confirmed the above hypothesis that along with penicillin, the degradation intermediates are also adsorbed on the surface activated carbons (Figure 6).

It appears that the confinement of pencillin molecules entrapped in the nanopores of the activated carbons and thus the contact with carbon surface accelerates their breakdown, if compared to penicillin degradation occurring in the bulk solution. This breakdown is more remarkable for the carbons of hydrophilic nature (W and WO), and it could be likely a result of the strong local $\mathrm{pH}$ fall inside the small pores of the carbonaceous adsorbents. In other words, by utilizing the pores of the carbon materials as some kind of nanoreactor, the acid-base enhanced degradation of penicillin is intensified and thus its breakdown (hydrolysis) is accelerated. The positive effect of confinement on catalytic reactions in the pores of nanometric dimensions have been recently reported [Zhou, 2008; Greaser, 2007]. 
Interestingly, large amounts of penilloic acid were also identified in the extract of WOH700, confirming the alkaline degradation of PEN inside the pores of hydrophobic carbons (although at a larger extent than breakdown from solution at alkaline $\mathrm{pH}$ ). Some other unknown compounds were also detected in the HPLC chromatograms, although at very low intensities, and might be the result of further degradation of penicillin and its derivatives [De Weck, 1960].

The existence of the variety of species adsorbed in the activated carbons after its exposure to penicillin was further confirmed by thermal analysis. DTG profiles are shown in Figure 7, along with those of the initial carbons before adsorption. Besides the peak related to the removal of water (ca. $100^{\circ} \mathrm{C}$ ) and the broad bands corresponding to the decomposition of the surface functionalities on each carbon (for $\mathrm{W}$ carbon also arising from thermal instability due to its low activation temperature), a single desorption peak centered between $200-400{ }^{\circ} \mathrm{C}$ can be distinguished for all carbons exposed to PEN, regardless their chemical composition and acid/base character. It should correspond to the desorption of PEN and its degradation intermediates retained in the pores. Given the similarity in the chemical formulae of PEN and its intermediates, it is reasonable to expect that they are adsorbed on the same sites of the carbon materials (i.e, microporosity) and thus they desorb in similar temperature ranges.

The results also suggest that the chemical reactions between the adsorbed species and the adsorbent surface do not take place (chemisorption). On the contrary, the chemical degradation of PEN seems to be a favorable mechanism inside the pores of activated carbons, and the new compounds formed in the pores are readily adsorbed. It is worth to mention that those new compounds arising from the degradation of penicillin do not have the therapeutic activity of the antibiotic [Deshpande, 2004], which is an important aspect for wastewater remediation.

Penicillin retention on activated carbons seems to take place following two different processes: physisorption and reactive adsorption. The physisorbed fraction is basically the uptake in the pores of the activated carbon, driven by dispersive interactions between the 
non-polar basal planes of the carbon and the aromatic rings of penicillin ( $\pi$ electroncontrolled or non-specific adsorption). Besides, reactive adsorption of PEN takes place in the confined nanopores (e.g. hydrolysis catalyzed by the carbon surface chemistry). Thus, whereas the first process depends on the porosity of the adsorbent, the second one relies on the chemical nature of either the carbon or the target probe (PEN), and provokes irreversible transformations of the adsorbate. Reactive adsorption induced in the presence of activated carbons has been reported in our previous works for organosulfur compounds [Ania, 2007]. As mentioned above, the series of activated carbons were chosen based on their chemical composition, and it appeared that their acidity was of special importance owing to the instability of PEN in acidic solutions. Therefore the performance of activated carbons for penicillin uptake is strongly related to their capability of promoting the degradation of the antibiotic by hydrolysis (reactive adsorption).

\section{CONCLUSIONS}

This work highlights the importance of activated carbon surface features for the reactive adsorption of penicillin from solution and its degradation. Activated carbons of acidic and basic nature promote the reactive adsorption of penicillin through the acid-base interactions between the antibiotic and the adsorbent surface which is followed by hydrolysis. This results in formation of a variety of degradation compounds which do not exhibit therapeutic activity. The uptake and reactive adsorption capability is strongly related to the chemical features of the adsorbents. Activated carbons of acidic nature present the lowest adsorption capacity of penicillin, although the rate of degradation of the adsorbed molecules is accelerated. In contrast, although basic carbons enhance the amount adsorbed the degradation occurs to lesser extent. Our results allowed the identification of two different mechanism of adsorption for penicillin from aqueous solution on activated carbons: physisorption in the microporous network, and reactive adsorption via hydrolysis of the PEN molecules adsorbed in the confined nanospace of the adsorbents. 


\section{Acknowledgements}

This work was supported by the Spanish Ministry of Science and Innovation, MICINN (project CTM2008-01956). COA thanks Dr. Vix from IS2M (France) for providing the TPD-MS. COA and TJB are grateful to the Spanish Council for Scientific Research (CSIC) for their Mobility Actions (grants PA1001122 and PA2001393).

\section{REFERENCES}

Aksu, Z., Tunc, O.: Application of biosorption for penicillin G removal: comparison with activated carbon, Process Biochem., 40, 831-847 (2005)

Al-Ahmad, A., Daschner, R.D., Kummerer, K.: Biodegradability of cefotiam, ciprofloxacin, meropenem, penicillin $\mathrm{G}$, and sulfamethoxazole and inhibition of waste water bacteria, Arch. Environ. Contain. Toxicol. 37, 158-163 (1999)

Ania, C.O., Menendez, J.A., Parra, J.B., Pis, J.J. : Microwave-assisted regeneration of activated carbons loaded with pharmaceuticals, Wat. Res. 41, 3299-3306 (2007)

Ania, C.O., Parra, J.B., Arenillas, A., Rubiera, F., Bandosz, T.J., Pis J.J.: On the mechanism of reactive adsorption of dibenzothiophene on organic waste derived carbons, Appl. Surf. Sci., 253, 5899-5903 (2007)

Arnott, I.A., Weatherley, L.R.: The stability of penicillin $G$ during recovery by electrically enhanced extraction, Process. Biochem. 30, 447-455 (1995)

Deshpande, A.D., Baheti, K.G., Chatterjee, N.R.: Degradation of $\beta$-lactam antibiotics, Current Science, 87, 1684-1695 (2004)

Bandosz, T.J., Ania C.O.: Surface chemistry of activated carbons and its characterization, in: T.J. Bandosz (Ed.), Activated Carbon Surfaces in Environmental Remediation, in the series Interface Science and Technology, 7, Elsevier, New York, 2006, pp. 159229.

Chaubal, M.V., Payne, G.F. Reynolds, C.H., Albright, R.L.: Equilibria for the adsorption of antibiotics onto neutral polymeric sorbents: experimental and modeling studies, Biotechnol. Bioeng., 47, 215-226 (2004)

Choi, K.J., Kim, S.G., Kim, S.H.: Removal of antibiotics by coagulation and granular activated carbon filtration, J. Haz. Mat. 15, 38-43 (2008)

Daughton, C.G., Ternes, T.A.: Pharmaceuticals and personal care products in the environment: Agents of subtle change?, Environ. Health Perspect. 107, 907-938 (1999) 
De Weck, A.L., Eisen, H.N.: Some immunochemical properties of penicillenic acid: an antigenic determinant derived from penicillin, J Experimental Medicine 112, 12281247 (1960)

Figueiredo, J.L., Pereira, M.F.R., Freitas, M.M.A., Orfao, J.J.M.: Modification of the surface chemistry of activated carbons. Carbon 37, 1379-1389 (1999)

Gomez, M.J., Martinez-Bueno, M.J., Lacorte, S., Fernandez-Alba, A.R., Aguera, A.: Pilot survey monitoring pharmaceuticals and related compounds in a sewage treatment plant located on the Mediterranean coast. Chemosphere, 66, 993-1002 (2007)

Gower, J.L., Risbridger, G.D., Redrup, M.J.: Positive and negative ion fast atom bombardment mass spectra of some penicilloic acids, The Journal of Antibiotics, 35 33-43 (1983)

Graeser, M., Pippel, E., Greiner, A., Wendorff, J.H.: Polymer Core-Shell Fibers with metal Nanoparticles as Nanoreactor for Catalysis, Macromolecules, 40, 6032-6039 (2007)

Hernando, M.D. Mezcua, M, Fernandez-Alba, A.R., Barceló, D.: Environmental risk assessment of pharmaceutical residues in wastewater effluents, surface waters and sediments, Talanta 69, 334-342 (2006)

Jones, O.A., Lester, J.N., Voulvoulis, N.: Pharmaceuticals: a threat to drinking water? Trends Biotechnol. 23, 163-167 (2005)

Kolpin, D.W., Furlong, E.T., Meyer, M., Thurman, E.M., Zaugg, S.D., Barber, L.B., Buxton, H.A.T.: Pharmaceuticals, hormones, and other organic wastewater contaminants in U.S. streams, 1999-2000: A national reconnaissance, Environ. Sci. Technol. 36, 1202-1211 (2002)

Li D., Yang M., Ju, J., Zhang, Y., Chang, H., Jin, F.: Determination of penicillin G and its degradation products in a penicillin products wastewater treatment plant and the receiving river, Water Research 42, 307-337 (2008)

Mestre, A.S., Pires, J., Nogueira, J.M.F., Parra, J.B., Carvalho, A.P., Ania C.O.: Wastederived activated carbons for removal of ibuprofen from solution: Role of surface chemistry and pore structure, Bioresour. Technol. 100, 1720-1726 (2009)

Ruiz, B., Cabrita, I., Mestre AS, Parra, J.B., Pires, J., Carvalho, A.P., Ania C.O.: Surface heterogeneity effects of activated carbons on the kinetics of paracetamol removal from aqueous solution Appl. Surf. Sci. 256, 171-5176 (2010)

Moats, W.A., Romanowski R.D.: Determination of Penicillin G in Beef and Pork Tissues Using an Automated LC Cleanup, J. Agric. Food Chem. 46 (1998) 1410-1413.

Noh, J.S., Schwarz, J.A.: Estimation of the point of zero charge of simple oxides by mass titration. J. Colloid Interface Sci. 130, 157-164 (1989) 
Robberson, K.A., Waghe1, A.B., Sabatini D.A., Butler E.C.: Adsorption of the quinolone antibiotic nalidixic acid onto anion-exchange and neutral polymers, Chemosphere, 63 934-941 (2006)

Schwartz, M.A.: Mechanism of degradation of penicillin G in acidic solution, J Pharm. Sci., 54, 472-473 (1965)

Zhou, L., Wang, S., Dai, W., Zhou, Y.: Intensified Reaction of Dilute Thiophenes in Nanoreactor, J. Phys. Chem. A, 112, 1887-1890 (2008) 
Figure 1. Molecular formula and selected chemical properties of penicillin G.

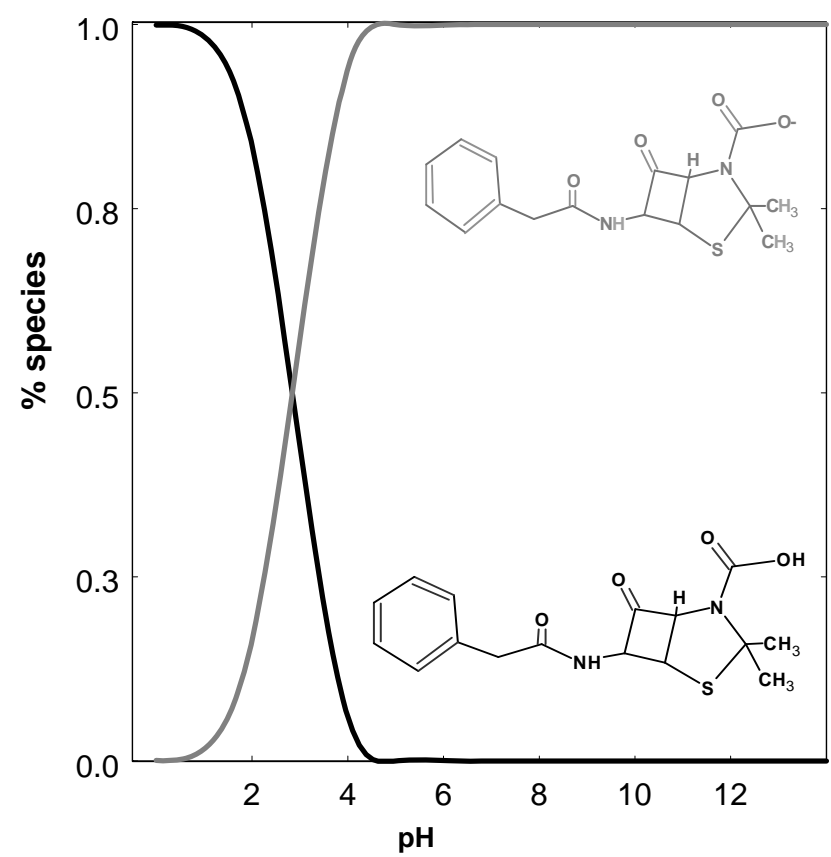

Penicillin $\boldsymbol{G}$

Molecular formula (sodium salt):

$\mathrm{C}_{16} \mathrm{H}_{17} \mathrm{~N}_{2} \mathrm{NaO}_{4} \mathrm{~S}$

Molecular weight: 356.37

Water solubility $(25$ ㅇ) $): 10 \mathrm{~g} / 100 \mathrm{~mL}$

pKa: 2.74

Log P(octanol water partition

coefficient): 1.5 


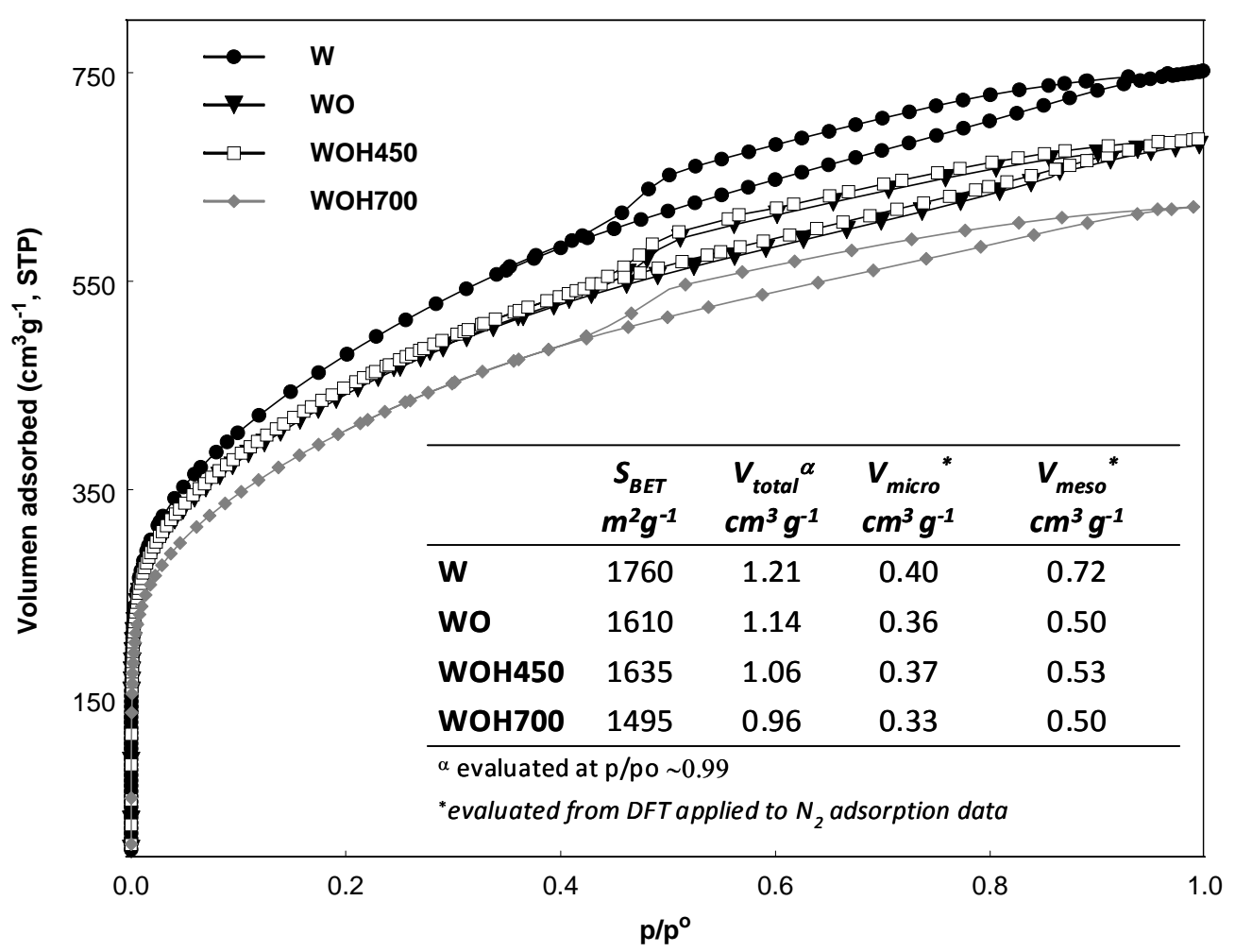

Figure 2. Nitrogen adsorption data of the studied carbons with indication of main textural parameters therefrom calculated. 

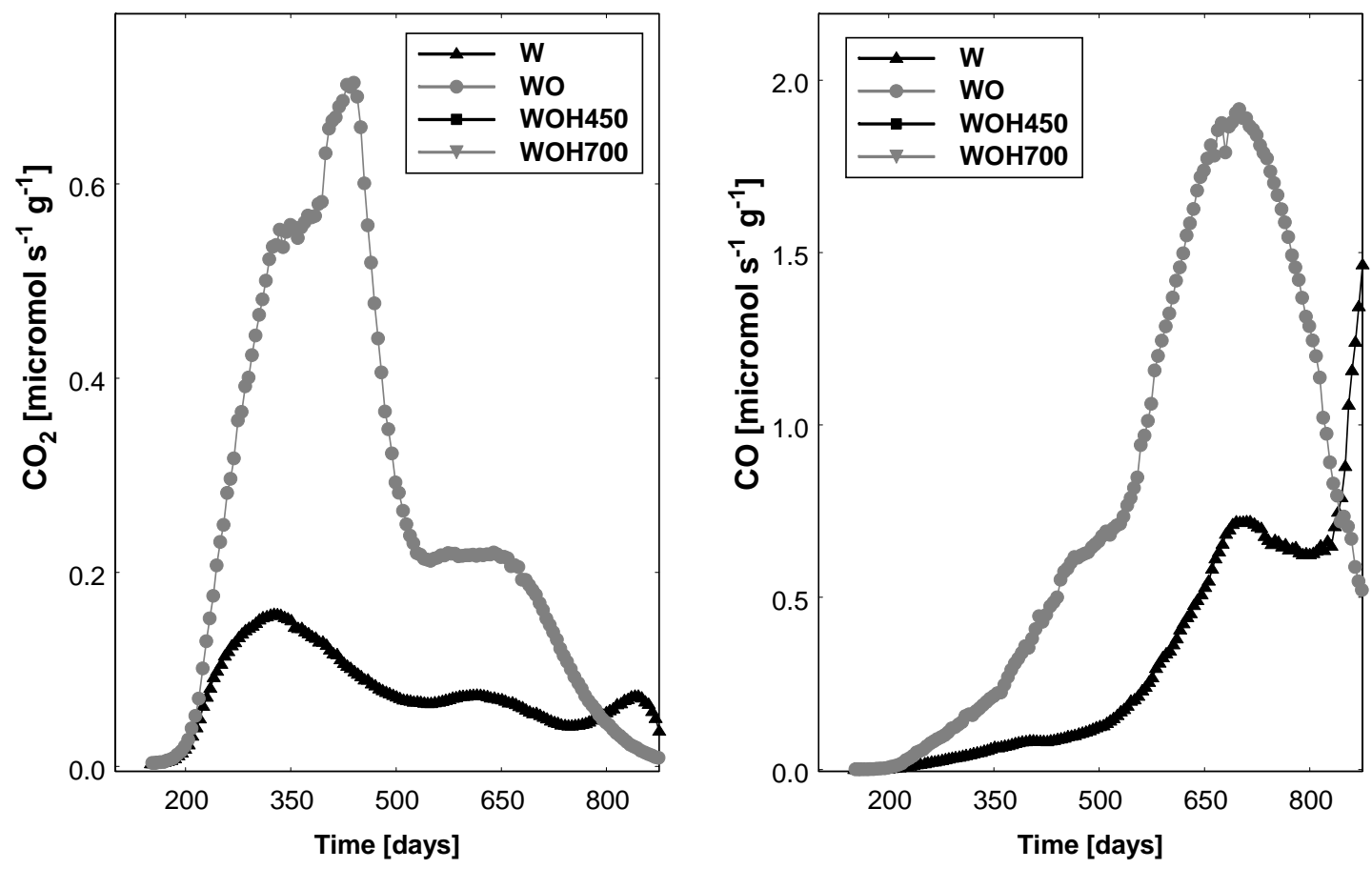

Figure 3. $\mathrm{CO}$ and $\mathrm{CO}_{2}$ profiles of the investigated carbons evaluated by TPD-MS. 

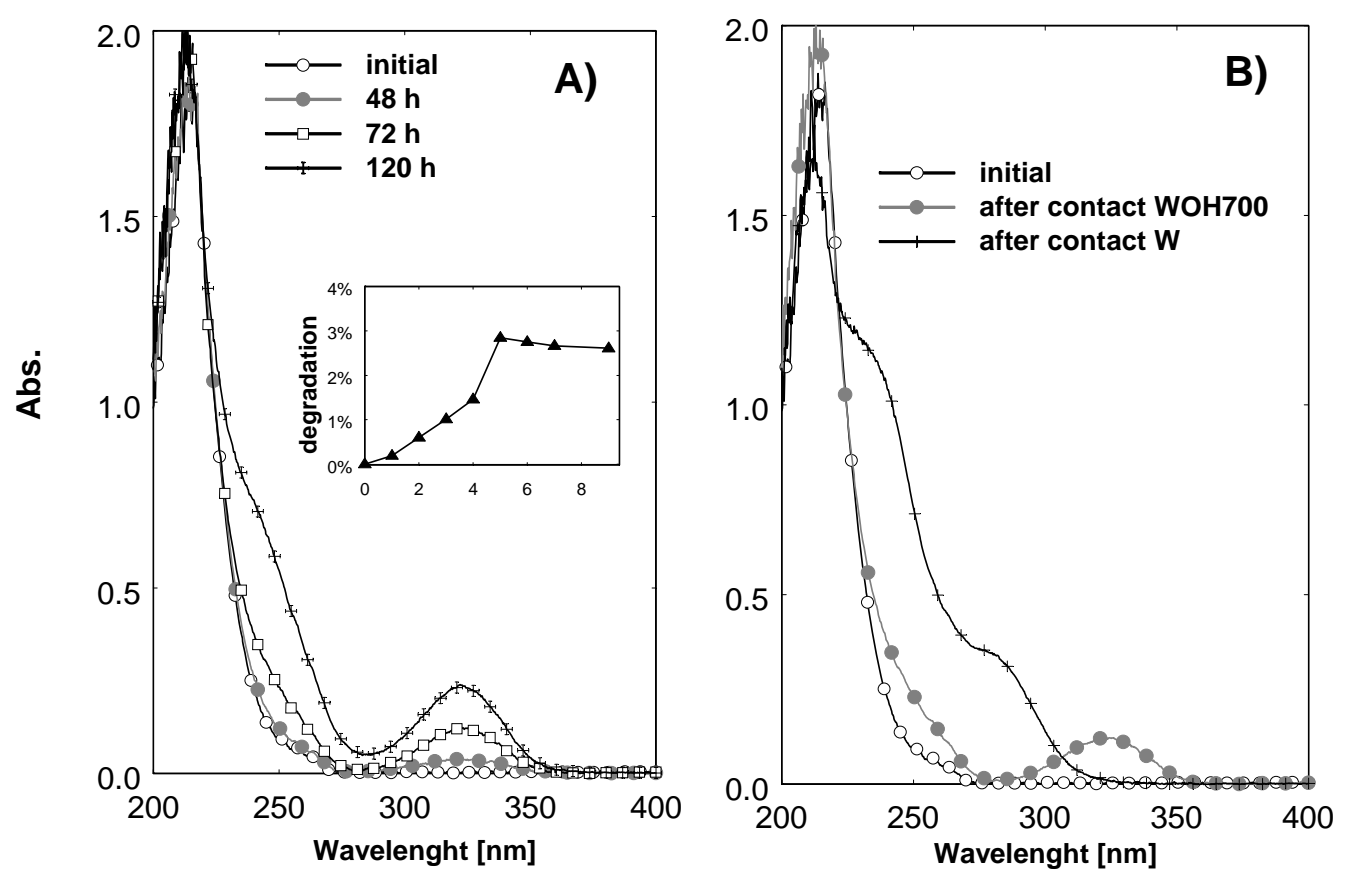

Figure 4. (A) Evolution of UV spectra of the initial penicillin solution (inset: degradation percentage upon time). (B) Changes in the UV spectra of the initial penicillin solution after contact with W and WPH700 carbons. 

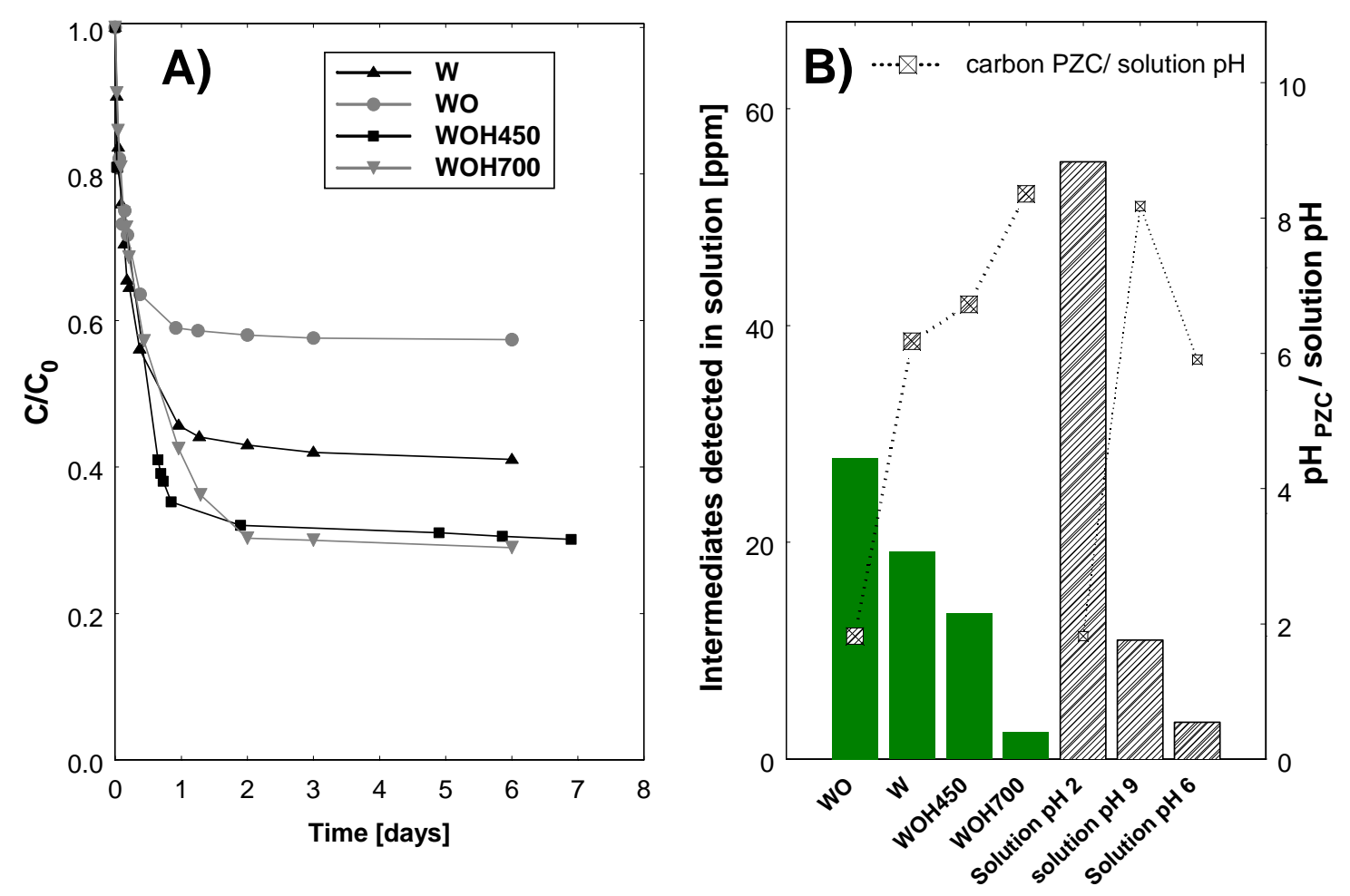

Figure 5. (A) Kinetics of penicillin disappearance from solution. (B) Quantification of penicillin degradation intermediates detected in solution after contact with the carbons and by effect of $\mathrm{pH}$. 


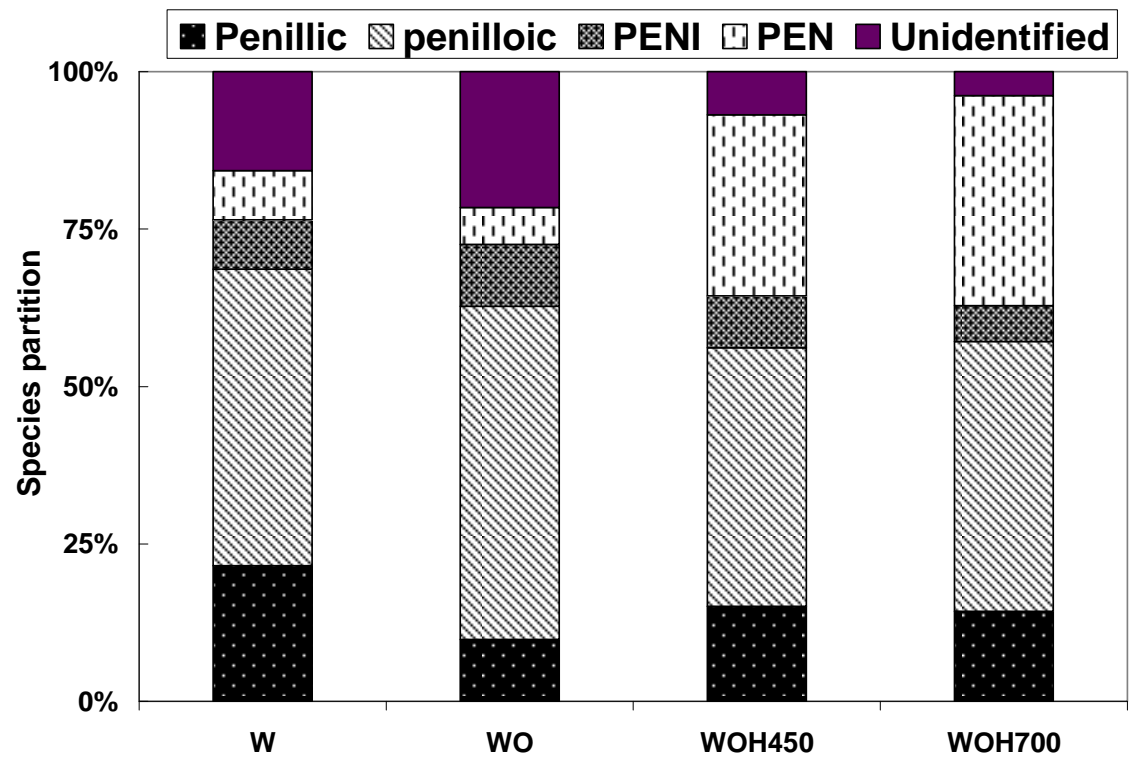

Figure 6. Distribution of penicillin and degradation intermediates retained inside the porosity of the studied carbons, retrieved after extraction of the loaded carbons. 

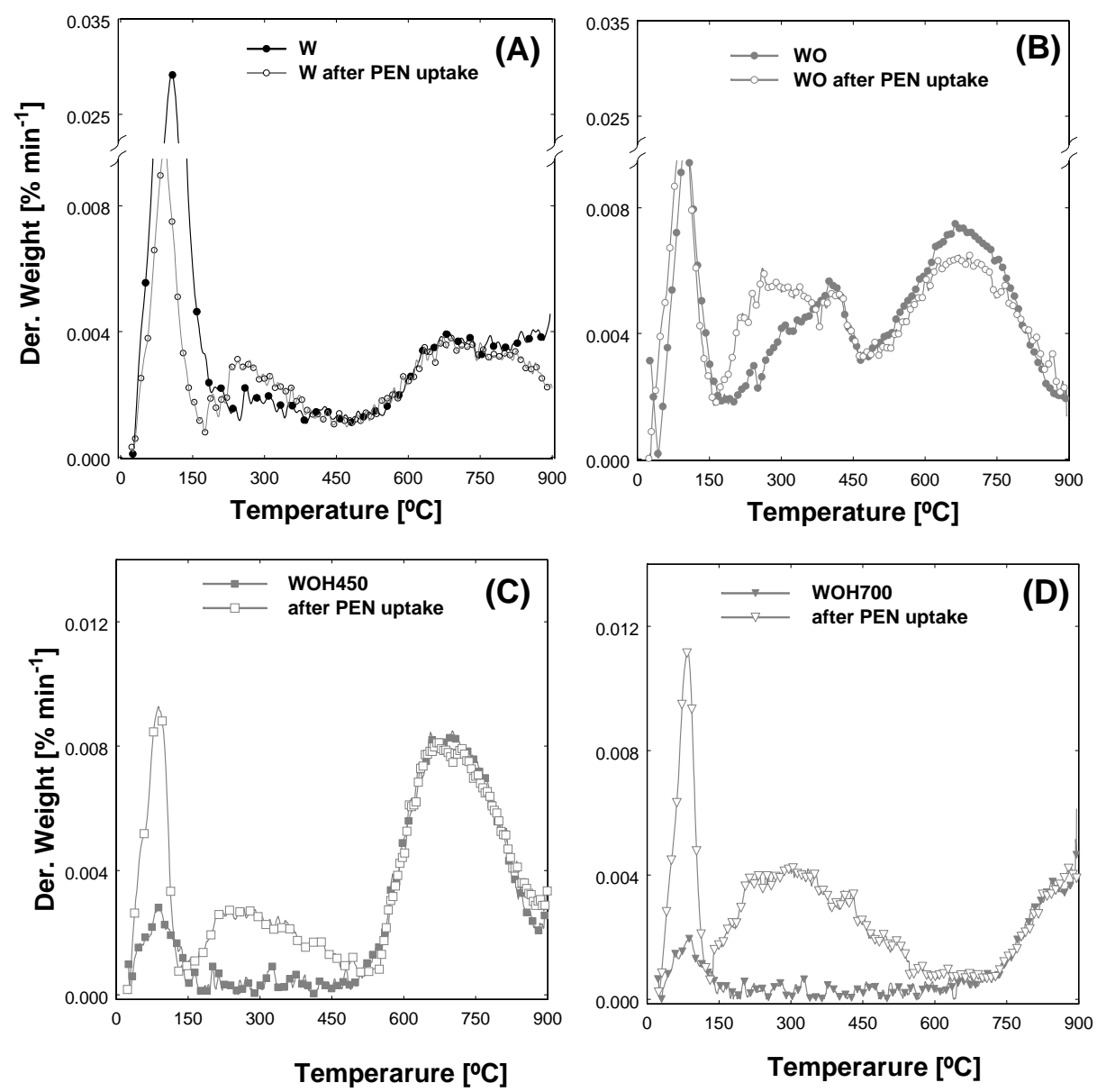

Figure 7. Desorption profiles (DTG) before and after penicillin uptake on the different carbons. 
Table 1. Chemical parameters of the investigated activated carbons.

\begin{tabular}{lcccccc}
\hline & $\mathbf{p H}$ PZC & $\begin{array}{c}\mathbf{O} \\
{[\mathbf{w t .} \%]}\end{array}$ & $\begin{array}{c}\mathrm{CO} \\
\mu \mathrm{mol} / \mathbf{g}\end{array}$ & $\begin{array}{c}\mathbf{C O}_{2} \\
\mu \mathbf{m o l} / \mathbf{g}\end{array}$ & $\begin{array}{c}\mathbf{H}_{2} \mathbf{O} \\
\mu \mathbf{m o l} / \mathbf{g}\end{array}$ & $\begin{array}{c}\text { Total } \\
\mu \mathbf{m o l} / \mathbf{g}\end{array}$ \\
\hline W & 6.8 & 5.1 & 1.79 & 0.37 & 1.56 & 2.37 \\
WO & 2.0 & 12.1 & 3.55 & 1.14 & 1.99 & 5.82 \\
WOH450 & 8.0 & 6.3 & 2.65 & 0.32 & 1.30 & 3.07 \\
WOH700 & 9.2 & 1.2 & 1.06 & 0.17 & 0.46 & 1.14 \\
\hline
\end{tabular}

